\title{
Sensorineural hearing loss as the first manifestation of Sjögren's syndrome
}

\author{
Raquel Sousa Almeida ${ }^{1 *}$, Ana Alves Oliveira ${ }^{1}$, Petra M. Pego ${ }^{1}$, Yahia Abuowda ${ }^{1}$, Iuri Gaspar ${ }^{2}$, João Matos Costa ${ }^{1}$

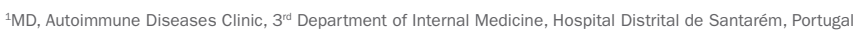 \\ ${ }^{2} \mathrm{MD}$, Otorhinolaryngology Department, Hospital Distrital de Santarém, Portugal \\ Article received: 5/29/2016 \\ Accepted for publication: 5/31/2016 \\ *Correspondence: \\ Hospital Distrital de Santarém, Serviço de Medicina III \\ Address: Av. Bernardo Santareno, 2005-177 \\ Santarém - Portugal \\ almeida.raquelsousa@gmail.com
}

\section{INTRODUCTION}

Dysfunction of inner ear, vestibulocochlear nerve or central brain processing centers leads to sensorineural hearing loss (SNHL). Autoimmune inner ear disease (AIED) is a rare but potentially treatable cause of hearing loss, characterized by progressive evolution over weeks to months. ${ }^{1}$ In 1958 , an antigen-antibody reaction was described in 13 patients with progressive bilateral SNHL, ${ }^{2}$ and later, in 1979, the term autoimmune SNHL was proposed after an idiopathic bilateral progressive SNHL had markedly improved with steroids therapy. ${ }^{3}$ Since there are no defined diagnostic criteria for AIED, it remains a diagnosis of exclusion, supported by clinical suspicion and responsiveness to corticotherapy. About one third of the cases of AIED is secondary to a systemic autoimmune disease and may, rarely, be the first manifestation of the latter. ${ }^{4,5}$

\section{Method}

Description of a clinical case, based on the data referred to in the clinical process.

\section{Results}

We present the case of a 65-year-old woman who attended the otorhinolaryngologist due to progressive and cumulative unilateral left hearing loss over one month, without any other accompanying symptoms. She had been diagnosed with breast cancer eleven years before, being successfully treated with chemotherapy and right mastectomy. She was medicated for arterial hypertension and hypothyroidism. Her son had been recently diagnosed with a cerebellopontine angle tumor. She had no history of vertigo or ocular problems. Otoscopy was normal but the Weber test lateralized to the right and the Rinner test were both positive. The neurological examination was normal, including no cerebellar or vestibular findings. The remaining physical test was unremarkable.
An audiogram was performed, with left hearing loss described as $-70 \mathrm{~dB}$ in the $8000 \mathrm{~Hz}$ range, confirming the suspicion of SNHL (Figure 1A). The routine laboratory workup and magnetic resonance imaging were normal.

She was started on $60 \mathrm{mg}$ of prednisolone $(1 \mathrm{mg} / \mathrm{kg} /$ day) with immediate response (Figure 1B). Steroid tapering was started and, after four weeks, she stopped the treatment. A relapse of symptoms occurred during the following month, with deterioration of hearing levels registered in the audiogram (Figure 1C). Corticotherapy was restarted and AIED was considered the most likely diagnosis, therefore she was referred to the autoimmune diseases clinic to exclude a systemic autoimmune disease.

She complained of dry eyes and mouth but denied arthralgia, parotid swelling or cold extremities, and had no history of recurrent abortion. The physical examination remained normal aside from mucosal dryness confirmed by the Schirmer test ( $<5 \mathrm{~mm}$ in 5 minutes). Laboratory tests showed normal liver and thyroid function, with positive thyroid peroxidase antibodies. Antinuclear antibodies were positive - she had anti-mitochondrial M2 antibodies but anti-Ro and anti-La where negative. Other autoimmune tests, including rheumatoid factor, anti-neutrophil cytoplasmic, anti-centromere, anti-neuronal antibodies, and lupus anticoagulant, were negative. She was observed by an ophthalmologist who reported no significant findings. The thyroid echography showed a microgranular pattern. She was treated with pilocarpine and a scintigraphy was performed, showing hypofunction of the salivary glands. The biopsy revealed significant lymphoid infiltrate - class III on the Chisholm Mason scale - confirming Sjögren's syndrome (SS).

After four months of low dose corticotherapy $(5 \mathrm{mg}$ of prednisolone), she had no symptoms of hearing loss and her left audiogram showed a $50 \mathrm{~dB}$ improvement (Figure 1D). 


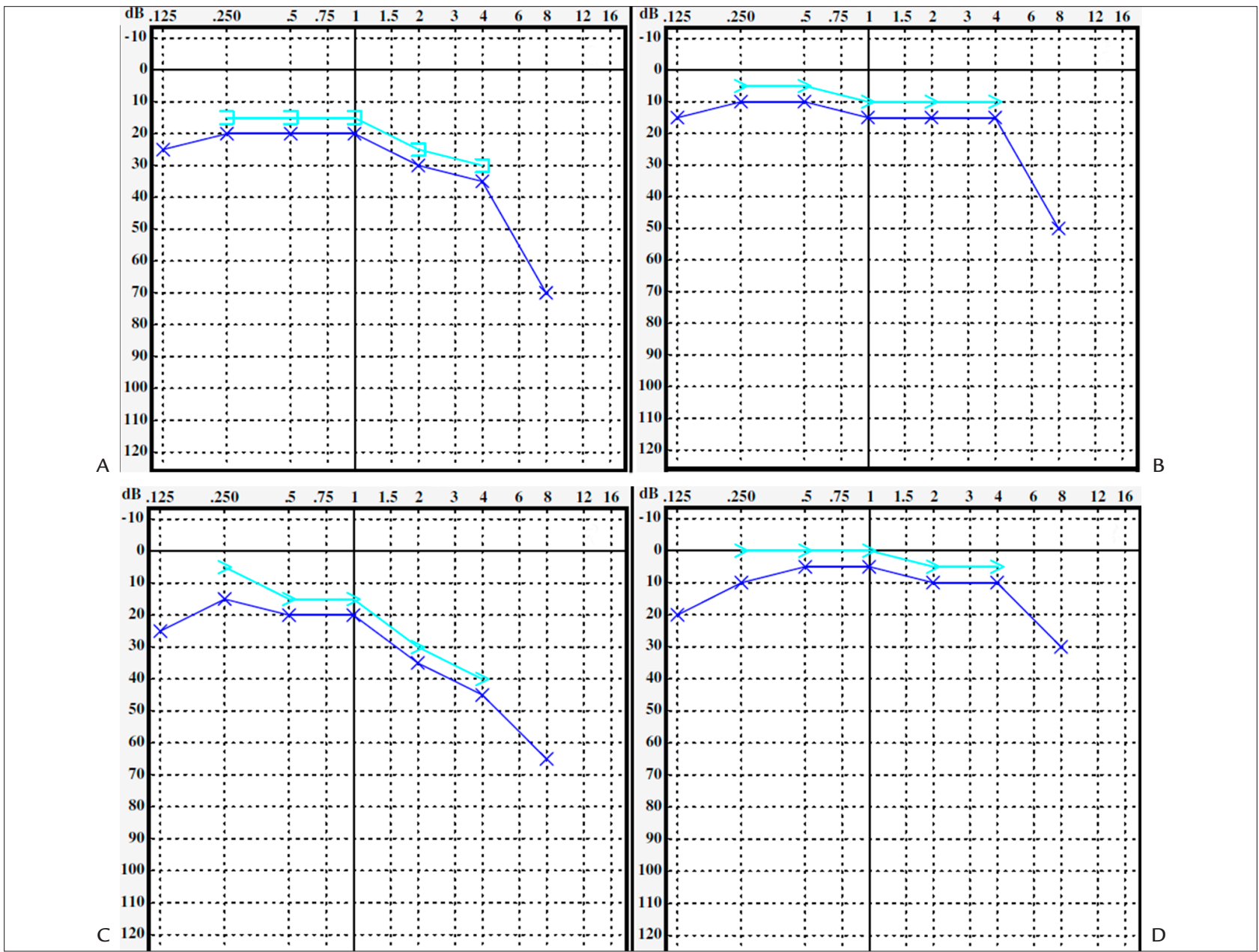

FIGURE 1 A. First audiogram, with left hearing loss described as $-70 \mathrm{~dB}$ in the $8000 \mathrm{~Hz}$ range, confirming the suspicion of SNHL. B. Audiogram on $60 \mathrm{mg}$ of prednisolone. C. Audiogram after stopping steroids, showing deterioration of hearing levels. D. Audiogram after four months of low dose corticotherapy, showing a $50 \mathrm{~dB}$ improvement.

\section{Discussion}

AIED is a SNHL which may have uni- or bilateral involvement and presents with progressive deterioration of auditory function over weeks to months. The time course is very important for the differential diagnosis between sudden hearing loss (hours to days) and presbycusis (years). It usually affects people in between the third and the sixth decade of life. Vestibular symptoms may be present., 3,5,6

SNHL can be the initial manifestation of a systemic autoimmune disease such as SS, systemic lupus erythematosus, granulomatosis with polyangiitis (Wegener's), ${ }^{7}$ polyarteritis nodosa, rheumatoid arthritis, and Cogan's syndrome. ${ }^{8,9}$ There is a significant association between thyroid autoimmunity and Ménière's disease, which can itself be very difficult to distinguish from AIED in the first months, ${ }^{10}$ but our patient had no vestibular symptoms. The relationship between SS and AIED was studied by Tumiati et al,, who suggested the performance of audiometric tests in patients with this syndrome. ${ }^{11}$ In this case, despite seronegative antiRo and anti-La, there were clinical, imaging, and histological findings that allowed the diagnosis of SS. Although antimitochondrial antibodies are characteristic of primary biliary cirrhosis, it is known that their presence in SS patients can occur, presenting a higher risk of liver involvement ${ }^{12}$ which increases the importance of surveillance by a multidisciplinary team. Systemic corticosteroids should be initiated as soon as possible since the prognosis is time dependent. ${ }^{5}$

\section{Conclusion}

This case, in which the medical findings led to the diagnosis of SS, illustrates the importance of a multidisciplinary approach and clinical suspicion of an autoimmune 
cause for progressive SNHL. After a response to corticotherapy, the possibility of an association with autoimmune systemic diseases should be thoroughly investigated.

\section{Key messages}

- Autoimmune inner ear disease is a diagnosis of exclusion, supported by clinical suspicion and responsiveness to corticosteroids.

- It is secondary to an autoimmune disease in one third of the cases.

- Although it is an unusual etiology of hearing impairment, it is important to recognize it, as early diagnosis and treatment can have a marked effect on the clinical outcome.

\section{Notes}

- No funding to declare.

- No animal or human studies were carried out by the authors for this article.

- The authors declare that none of the presented data allows for the identification of the patient.

\section{Conflict OF InTERest}

The authors declare no conflict of interest.

\section{RefEREnCES}

1. Bovo R, Aimoni C, Martini A. Immune-mediated inner ear disease. Acta Otolaryngol. 2006; 126(10):1012-21.

2. Lehnhardt E. [Sudden hearing disorders occurring simultaneously or successively on both sides]. Z Laryngol Rhinol Otol. 1958; 37(1):1-16.

3. McCabe BF. Autoimmune sensorineural hearing loss. Ann Otol Rhinol Laryngol. 1979; 88(5 Pt 1):585-9.

4. Hughes GB, Kinney SE, Barna BP, Calabrese LH. Practical versus theoretical management of autoimmune inner ear disease. Laryngoscope. 1984; 94(6):758-67.

5. Mijovic T, Zeitouni A, Colmegna I. Autoimmune sensorineural hearing loss: the otology-rheumatology interface. Rheumatology (Oxford). 2013; 52(5):780-9.

6. Boulassel MR, Deggouj N, Tomasi JP, Gersdorff M. Inner ear autoantibodies and their targets in patients with autoimmune inner ear diseases. Acta Otolaryngol. 2001; 121(1):28-34.

7. Scalcon MRR, Pereira IA, Rachid Filho A, Paiva ES. Manifestação otológica localizada em paciente com granulomatose de Wegener. Rev Bras Reumatol. 2008; 48(4):253-5.

8. Lunardi C, Bason C, Leandri M, Navone R, Lestani M, Millo E, et al Autoantibodies to inner ear and endothelial antigens in Cogan's syndrome. Lancet. 2002; 360(9337):915-21.

9. Harris JP, Sharp PA. Inner ear autoantibodies in patients with rapidly progressive sensorineural hearing loss. Laryngoscope. 1990; 100(5):516-24.

10. Fattori B, Nacci A, Dardano A, Dallan I, Grosso M, Traino C, et al. Possible association between thyroid autoimmunity and Menière's disease. Clin Exp Immunol. 2008; 152(1):28-32.

11. Tumiati, B, Casoli P, Parmeggiani A. Hearing loss in the Sjögren syndrome. Ann Intern Med. 1997; 126(6):450-3.

12. Maślińska M, Przygodzka M, Kwiatkowska B, Sikorska-Siudek K. Sjögren's syndrome: still not fully understood disease. Rheumatol Int. 2014; $35(2): 233-41$. 